\title{
DESIGN AND PERFORMANCE ANALYSIS OF SMART ROOF CLOTHESLINE SYSTEM BASED ON MICROCONTROLLER BY SMARTPHONE APPLICATION
}

\author{
Dewi Rasni Putri ${ }^{1}$, Doan Perdana ${ }^{2}$, Yoseph Gustommy Bisono ${ }^{3}$ \\ ${ }^{1,2,3}$ School of Electrical Engineering, Telkom University \\ 1'dewirasniputri95@gmail.com, ${ }^{2}$ doanperdana@ telkomuniversity.ac.id, ${ }^{3}$ bisono@telkomuniversity.ac.id
}

\begin{abstract}
Drying clothes is a simple activity to do at home and can not be avoid in daily life. The busyness for each individual outside home makes the activity is not simple anymore. The occurrence of problems such as rain, resulting clothes that have been dry become wet again. To avoid these conditions, the owner needs to be at home when the rain occurs. Smart home is a concept that can be the right and effective solution for every problems in the house. The ability of smart home to control almost all equipment at home make the activities at home efficiently. This Research develop a smart clothes roofing system that can be controlled and monitored by users through an application on smartphone. This system uses rain sensor, humidity and temperature sensors (DHT22), and light sensor (LDR). NRF24L01 as a transceiver module, Arduino Uno as microcontroller and Raspberry $\mathrm{Pi}$ as gateway to VPS. The test results obtained the maximum range of NRF2L01 to communicate is 97 meters in LOS (Line of Sight) condition. Distance range of NRF24L01 can also affected the delay and throughput in data transmission process. The availability and reliability of the system are $\mathbf{9 8 . 2 7 6 \%}$ and $\mathbf{9 8 . 2 6 4 \%}$.
\end{abstract}

Keywords: smart home, smart clothes roof system, microcontroller, application, smartphone

\section{Introduction}

The rapid development of telecommunications triggered the new various concepts that will be developed to utilizing the internet technology . One of the most popular concept is Internet of Things (IoT). IoT or Internet of Things is a concept that aims to expand the benefits of internet connectivity that is connected continuously $[1,2]$.

The application of IoT that is being developed is smart home. Smart home is a home equipped with high technology that allows various systems and devices at home to communicate with each other. Smart home system can be used to control almost all equipment in the house that the command can be sent with a remote control (remote) [3]. The application of smart home is easy and efficient to solve the many problems and activities at home, considering the various activities every individual person outside home. [3]

Household activities such as washing, drying clothes, cooking, cleaning the house is a simple activity that can not be released in everyday life. However, with the busyness of every individual that takes a lot of time outside the home makes the activity is not a simple anymore [4,5].

Just as when hanging clothes, the occurrence of problems such as rain, can make a person unaware and can not control the clothes that have been dry become wet again because of the rain [6]. This research develops a smart clothes roofing system adopt from smart home concept as one of solution to the problem in drying clothes. This system can be controlled and monitored by users through an application on smartphone. So it is expected to help the drying clothes that looks simple to be very easy and efficient.

\section{Smart Roof Clothesline System}

Smart roof clothesline system is a roofing system that can open or close the roof while rain ocurrences. The roof can monitored and controlled by user using a smartphone. This system adopt from smart home concept where to implemented this system need some hardware and software. Performance analysis is needed to know the quality of the system that has been implemented.

\subsection{Smart Home}

Smart home system is an application system that is a combination of technology and services devoted to the home environment with certain functions aimed at providing comfort, convenience, energy saving and security for the inhabitants. Intelligent home systems typically consist of control devices, monitoring and automation of some devices or home appliances that can be accessed through websites using both laptops and smartphones [6].

The intelligent home system consists of several supporting components that interact with each other. A house can be said to be a smart home if it has the internal network, intelligent control and home automation. A variety of smart home system applications with various features as future home concepts. The created applications are distinguished in terms of each function and purpose. 


\subsection{Hardware}

\subsubsection{Arduino Uno}

Programming used in Arduino is a simplified C language using Arduino libraries. Arduino also simplifies the process of working with a microcontroller, while offering a variety of advantages such as cheap, simple, easy programming, and it is open source [7].

\subsubsection{Rain Sensor}

Rain sensor is a sensor that can detect the occurrence of rain based on water that falls on the sensor panel. The rain sensor working principle is at the time of rain on the sensor panel, there will be electrolysis process by the rain water. It happens because of the rain water included into the electrolyte liquid where it can conduct electrical current, although very small and this process will active the relay in the sensor module [9].

\subsubsection{NRF24L01}

The Wireless Module NRF24L01 is a remote communications module that utilizes the ISM (2.4GHz) RF Radio Band (Industrial, Scientific and Medical). This module uses the SPI interface to communicate. The working voltage of this module is $3,3 \mathrm{~V}$ DC. This module can be used for PC peripherals, game tools, fitness, sports devices, children's toys and other tools $[11,12]$.

\subsubsection{Light Dependent Resistor (LDR) Sensor}

LDR (Light Dependent Resistor) is a type of resistor commonly used as a light detector or a light conversion measurement. Light Dependent Resistors, consisting of a semiconductor disk have two electrodes on its surface. However, because the response to light is quite slow, LDR is not used in situations where the intensity of light changes drastically. This sensor will change its resistance value if there is a change in the brightness of the light [13].

\subsubsection{DHT22 Sensor}

DHT22 is a digital sensor that can measure the temperature and humidity of the surrounding air. This sensor has excellent stability rates as well as highly accurate calibration features. The calibration coefficients are stored in the OTP program memory. So, when the internal sensor detects something, then this module includes the coefficients in its calculations. DHT22 includes the highest quality sensors, judged by response, fast data readability, and anti-interference capabilities [14].

\subsubsection{Raspberry Pi 3 Model B}

Raspberry Pi, often abbreviated as Rasp- $\mathrm{Pi}$, is a single-board circuit (SBC) computer that can be used to run office programs, computer games, and as media players to high-resolution video [15].

\subsection{Software}

\subsubsection{Domoticz}

Domoticz is used to convigure the devices in the system such as rain sensor, temperature and humadity sensor, light sensor, and motor servo. It is use to automation, control and monitor the data system in Raspberry Pi. The system is designed to operate in various operating systems. Domoticz is compatible with all browsers [16].

\subsubsection{MQTT}

MQTT stands for Message Queuing Telemetry Transport. MQTT is an open source connectivity protocol M2M/(IoT) -based connectivity (IoT) designed for limited devices and can work with low bandwidth, high latency or can runs on networks that are not reliable. MQTT is ideal for connected devices and mobile applications in the M2M / IoT era where bandwidth and battery power are the main considerations $[17,18]$.

\subsubsection{Android Studio}

Android Studio is an IDE for Android Development that is a development of Eclipse IDE, and is based on the popular Java IDE, the IntelliJ IDEA. Android Studio is the official IDE for Android app development. Android Studio has many new features compared to Eclipse IDE [19].

\subsection{Parameter Analysis}

\subsubsection{Throughput}

Throughput is the average speed of data received by a node within a given time interval. Throughput is the actual bandwidth at that moment. Its units are equal to the bandwidth of bits per second. Formula (1) is used for calculating the throughput value.

$$
\text { Throughput }=\frac{\text { Amount of data sent }}{\text { Time of data transmission }}
$$

\subsubsection{Delay}

Delay is the length of time required by data or information to get to the destination. 


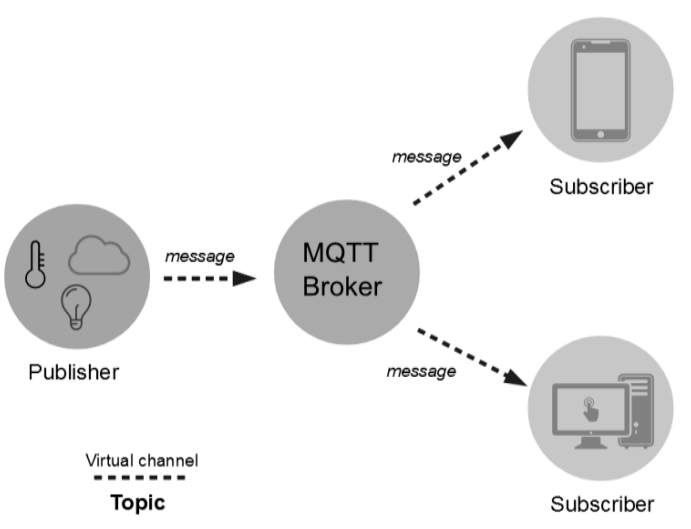

Figure 1. MQTT

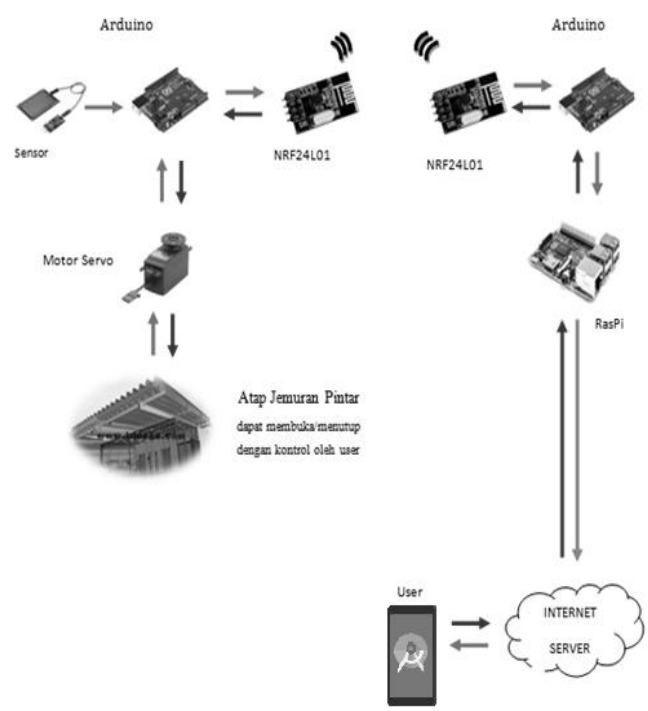

Figure 2. Model System

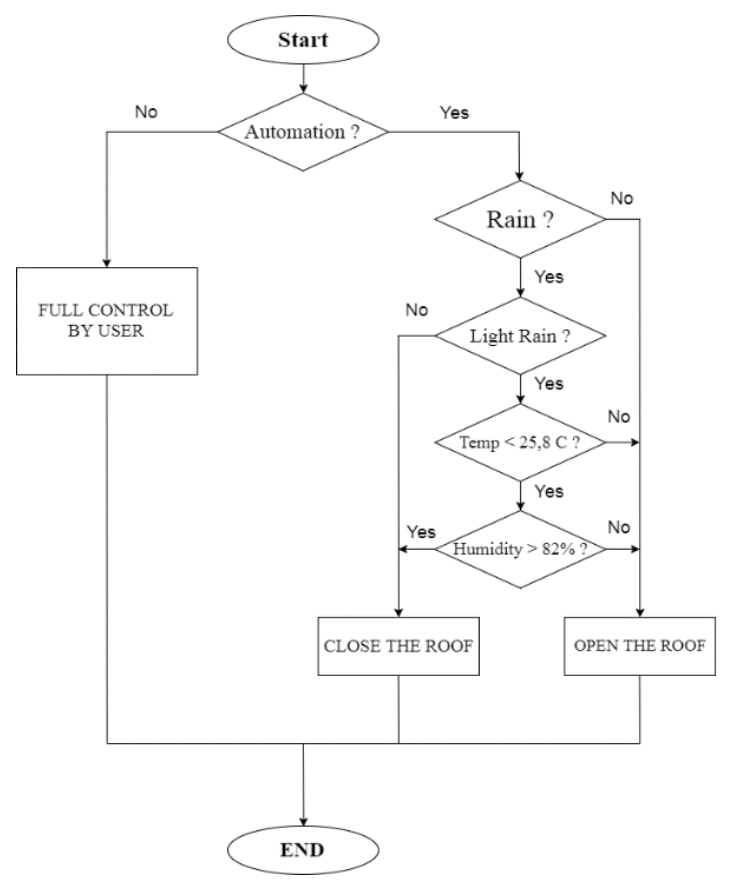

Figure 3. Work Flow System

\subsubsection{Reliability}

Reliability is the possibility of a system or component to be able to meet the required function, under certain conditions and at certain period.

$$
\text { Reability }=\frac{(\text { Uptime }- \text { Downtime })}{\text { Uptime }} \times 100 \%
$$

\subsubsection{Availibility}

Availability is the possibility of a system or component ready to operate when needed.

$$
\text { Availability }=\frac{\text { Uptime }}{(\text { Uptime }+ \text { Downtime })} \times 100 \%
$$

\section{Design and Workflow Model System}

The devices that used to create a smart roof clothesline system consists of sensors (rain sensor, temperature and humidity sensor, light sensor), NRF24L01, servo motor, Arduino, and Raspberry PI. There are two process in this system, they are monitoring process and controlling process. Monitoring process is sending the data of sensor and roof status to the user, while the controlling process in the form of commands that do by user to close or open the roof using a smartphone. In the Arduino microcontroller system will be connected with sensors such as rain sensor, temperature and humidity sensor, light sensor.

The Arduino will also be connected to the servo motor as a roof drive and also the NRF24L01 as a wireless communication module. On a local server of Raspberry Pi will also be connected with the NRF24L01 which is connected with Arduino. Internet server is used for the system that has been built to connect by user using android smartphone. So users can do monitoring and control using Android app.

\section{Evaluation and Analysis}

\subsection{LOS (Line of Sight) Condition}

\subsubsection{Range Of NRF24L01}

The maximum range of NRF24L01 could send data at a distance of 97 meters. The optimum distance for sending data from NRF24L01 to NRF24L01 is at a distance of $10-30$ meters where there is only a maximum of 1 data transmission failure. At a distance of 98 meters, the data was sent 30 times, but only 2 data received by the NRF24L01 receiver, the rest of the data failed to be received (RTO).

So it can be concluded that the maximum range of NRF24L01 to communicate at LOS condition at 97 meters. 


\subsubsection{Delay}

The result of delay testing on the LOS condition has increased the delay value significantly. The largest delay value at 97 meters with 4.358 milliseconds, indicating that the further the range between the NRF24L01 transmitter and the NRF24L01 receiver the resulting delay value will be greater. After the calculation of the delay from 10 meters to 97 meters obtained the average increase in the delay of each meter is 0.0357 millseconds per meter.

\subsubsection{Throughput Monitoring and Throughput Monitoring Controlling}

In the table above, we can see the decrease in throughput value with increasing distance. In testing the throughput value of monitoring at a distance of 40 meters an increase in throughput value with 4,842 Kbytes/sec.

While the value of throughput monitoring and controlling increased value at a distance of 50 meters and 60 meters. After the calculation of throughput value from 10 meters to 97 meters, the average value of throughput reduction of each meter is 46,198 bytes/sec, while the average of indirect decrease throughput monitoring and controlling every meter is $0.221 \mathrm{byte} / \mathrm{sec}$.

\subsection{Non LOS (Non Line Of Sight) Condition}

\subsubsection{Range Of NRF24L01}

The result of experiment of NRF24L01 communication in Non LOS condition with room and door as the obstacle, the maximum range of NRF24L01 can perform data transmission at the front of 306 room. In the test on the room terrace 307.30 data is sent, but all data is not received by NRF24L01 (RTO).

On the terrace room 307 (15 meters in non LOS condition) was not able to connect due to the obstacle in the form of wall room 307.

\subsubsection{Delay}

The result of NRF24L01 delay measurement under Non-LOS condition, constant delay value increased from 0.899 millisecond to 3.12 millisecond with increasing test distance. This shows the further distance between NRF24L01 then the data transmission process will take a longer time.

After the calculation of the delay from 6-points experiment then got the average increase of delay each point is 0.3702 millisecondd per room.

\subsection{QoS System on Internet Server}

\subsubsection{Monitoring Server}

- Delay

From the results of the test the average value of delay on the server obtained in 30 experiments is equal to 0.00922 seconds or equivalent to 92 milliseconds. This shows a very good delay index referring to ITU-T G.1010 which states that the delay value in very good category is less than 150 milliseconds.

\section{- Throughput}

The calculation of throughput value of server monitoring with 30 experiments, the average throughput of monitoring of 5440,919 bytes/sec or by $5.44 \mathrm{Kbytes} / \mathrm{sec}$. The average total packet delivered is 352 bytes with an average shipping time of 0.06513 seconds.

\subsubsection{Throughput Monitoring And Throughput Monitoring Controlling}

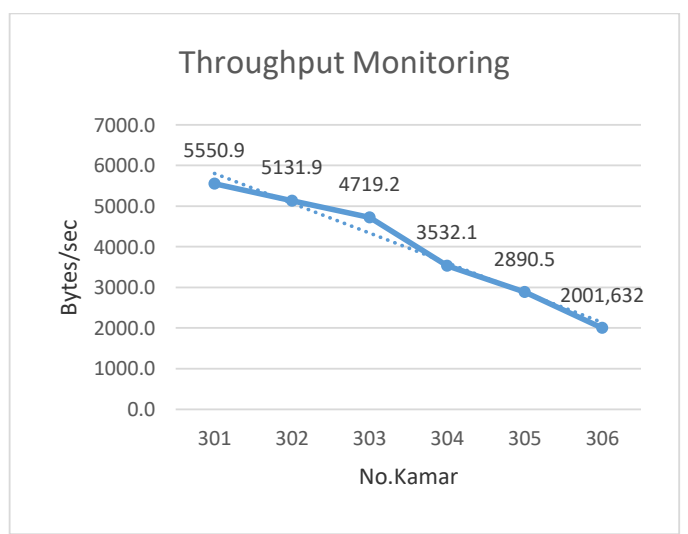

Figure 4. Throughput Monitor

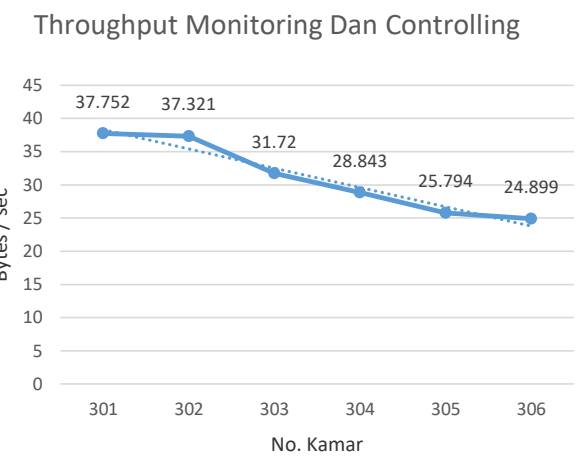

Figure 5. Throughput Monitoring and Controlling 


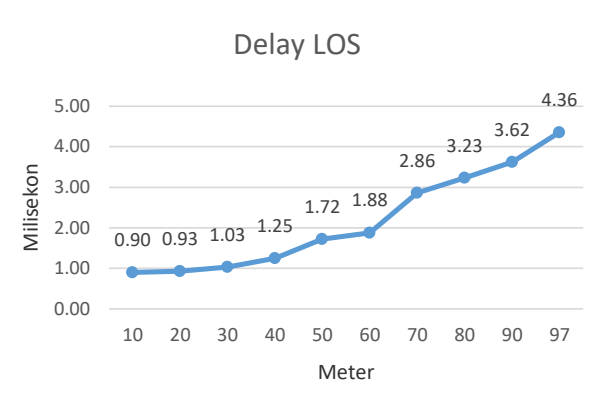

Figure 6. Delay LOS

Table 1. Range of NRF24L01

\begin{tabular}{|c|c|}
\hline Range (meter) & STATUS \\
\hline 10 & CONNECTED \\
\hline 20 & CONNECTED \\
\hline 30 & CONNECTED \\
\hline 40 & CONNECTED \\
\hline 50 & CONNECTED \\
\hline 60 & CONNECTED \\
\hline 70 & CONNECTED \\
\hline 80 & CONNECTED \\
\hline 90 & CONNECTED \\
\hline 97 & CONNECTED \\
\hline 98 & NOT CONNECTED \\
\hline
\end{tabular}

Table 2. Throughput

\begin{tabular}{|c|c|c|}
\hline \multicolumn{3}{|c|}{ THROUGHPUT } \\
\hline $\begin{array}{c}\text { Distance } \\
\text { (meter) }\end{array}$ & $\begin{array}{c}\text { T. Monitoring } \\
\text { (Bytes/sec) }\end{array}$ & $\begin{array}{c}\text { T. Monitoring and } \\
\text { Controlling (Bytes/sec) }\end{array}$ \\
\hline 10 & 5475,984 & 37,973 \\
\hline 20 & 4859,850 & 35,410 \\
\hline 30 & 4356,181 & 33,223 \\
\hline 40 & 4842,431 & 32,878 \\
\hline 50 & 3056,478 & 35,502 \\
\hline 60 & 2923,964 & 36,287 \\
\hline 70 & 1618,950 & 31,586 \\
\hline 80 & 1386,263 & 30,745 \\
\hline 90 & 1138,481 & 27,684 \\
\hline 97 & 991,761 & 23,329 \\
\hline
\end{tabular}

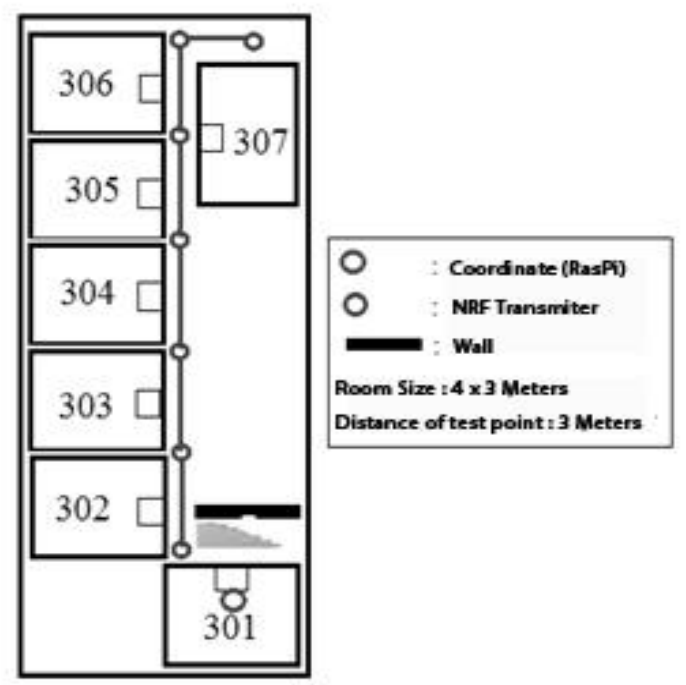

Figure 7. The Sketch Location ff Non Los Condition
Table 3. Range of NRF24L01

\begin{tabular}{|c|c|}
\hline No. Kamar & STATUS \\
\hline Front 301 & CONNECTED \\
\hline 302 & CONNECTED \\
\hline 302 & CONNECTED \\
\hline 304 & CONNECTED \\
\hline 305 & CONNECTED \\
\hline 306 & CONNECTED \\
\hline Terrace 307 & NOT CONNECTED \\
\hline
\end{tabular}

\section{Delay Non LOS}

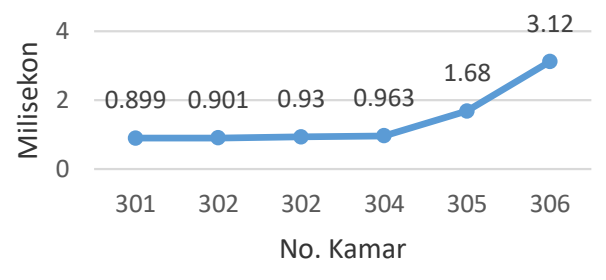

Figure 8. Delay NON LOS

Table 4. Reliability and Availibility

\begin{tabular}{|c|c|c|c|c|c|}
\hline \multicolumn{7}{|c|}{ RELIABILITY AND AVAILABILITY } \\
\hline $\begin{array}{c}\text { Uptime } \\
(\mathrm{sec})\end{array}$ & $\begin{array}{c}\text { Downtime } \\
(\mathrm{sec})\end{array}$ & $\begin{array}{c}\text { Availability } \\
\text { based on } \\
\text { time (\%) }\end{array}$ & $\begin{array}{c}\text { Reliability } \\
\text { based on } \\
\text { time (\%) }\end{array}$ & $\begin{array}{c}\text { Availability } \\
\text { based on } \\
\text { data (\%) }\end{array}$ & $\begin{array}{c}\text { Reliability } \\
\text { based on } \\
\text { data (\%) }\end{array}$ \\
\hline 886,893 & 15,556 & 98,2762 & 98,246 & 96,945 & 96,848 \\
\hline
\end{tabular}

\subsubsection{Controlling Server}

\section{- Delay}

The average result of delay controlling server measurement equal to 0.07564 second or equal to 75,64 millisecond in 30 times experiment. The value of server controlling delay is still included in the very good delay index referring to ITU-T G.1010.

\section{- Throughput}

From the test results throughput server controlling value obtained by the average throughput is 7770.017 bytes / sec or 7.77 Kbytes / sec. With the average size of packets sent is 221.5 bytes, while the average delivery time is 0.0287 seconds. The value of throughput controlling obtained is greater than the value of throughput monitoring due to the value of delivery time or timespan smaller than the timespan performed on the monitoring process. This is in accordance with the theory that the smaller the time taken in the delivery of data the greater the value of throughput to be generate

\subsubsection{Monitoring And Controlling Server}

\section{- Throughput}

The process of monitoring and controlling the server is the process of sending data from Raspberry 
Pi to Android users and vice versa. Data throughput is taken on the VPS Wireshark application and is accomplished by retrieving one monitoring data and one data controlling by performing 30 experiments. From the experimental data the average throughput is 2476,492 bytes/sec or 2,476 Kbytes/sec. While the average package size is 783.3 bytes with the average delivery time is 0.6211 seconds. From the results obtained, the throughput value in the monitoring and controlling process is the smallest compared with the throughput value in the monitoring process and the controlling process only. It is caused by the length of time required to perform the process of transmitting monitoring and controlling data at once, thus causing small throughput values in the monitoring process and controlling the server.

\subsection{Reliability and Availibility}

In this test is done by sending data as much as 491 data in 902,449 seconds or equal to 15 minutes 2,499 seconds. The value of availibility and reability of the system can be reviewed into two parts based on time and based on the number of data. In the data transmission process there are 15 data failed to transmit with a total time of 15.556 seconds.

The amount of data or the amount of time that fails is the value of downtime on the system. While the uptime is the total time of successful delivery data of 886.893 seconds with the number 476 data. So as to produce the value of availibility of $98.2762 \%$ and reliability value of $98.2246 \%$ in the observation of time units. And also availability value of $96.945 \%$ and reliability value of $96.848 \%$ in observation of a lot of data.

\section{Conclusion}

From the results that have been obtained after the testing, it can be concluded as follows:

1. Prototype smart clamping roofing system that has been made to work properly and function in accordance with the specified.

2. Prototype smart clamping roofing system that has been made can be done by monitoring by displaying parameters of rainfall, temperature, humidity, light, and roof status.

3. Prototype clever roofing system that has been made can be done by the user's controlling using a smartphone in the form of closing the roof or opening the roof.

4. The maximum working range of NRF24L01 under LOS (Line of Sight) is at 97 meters.

5. On testing the network quality of NRF24L01, the smallest delay value is located at 10 meters of 0.899 milliseconds, and the largest delay is at 97 meters with a value of 4.358 milliseconds.

6. In testing, the network quality of NRF24L01, the largest throughput value is 5475,984 bytes/sec at 10 meters distance, and the smallest throughput value is 994,764 bytes/sec at 97 meters.

7. In testing, the quality of the network server in 30 experiments, obtained the average delay monitoring value of 92 milliseconds and the average delay controlling value of 75.64 milliseconds.

8. On testing of server network quality in 30 experiments, the average value of the throughput of monitoring was 5.44 Kbytes/sec and the average throughput controlling of 7.77 Kbytes/sec, while the throughput of monitoring and controlling 2,476 Kbytes/sec.

9. The value of availability on the whole system is $98.2762 \%$, while the value of system reliability is $98.2246 \%$.

\section{References}

[1] Rouse, "Internet of Things (IoT)," WhatIs, p. 6, 2014.

[2] International Telecommunication Union, "Overview of the Internet of things," Ser. Y Glob. Inf. infrastructure, internet Protoc. Asp.

[3] next-generation networks - Fram. Funct. Archit. Model., p. 22, 2012.

[4] A. Homechat and T. Smarthome, "HomeChat: a way communicate with home instrument," 2011.

[5] S. Amalia, "Intisari Jemuran Pintar Berbasis Arduino Dengan Sensor Hujan Abstract Smart Laundry hanger prototype based on arduino uno with rain."

[6] M. T. Industri et al., "Perancangan jemuran otomatis pendeteksi hujan," pp. 197-203, 2011.

[7] F. G. ADITYA, “ANALISIS DAN PERANCANGAN PROTOTYPE SMART HOME DENGAN SISTEM CLIENT SERVER BERBASIS PLATFORM ANDROID MELALUI KOMUNIKASI WIRELESS,” p. 2015, 2015.

[8] A. Uno and R. Front, "Arduino Uno."

[9] http://henrysbench.capnfatz.com/henrysbench/arduino-sensors-and-input/arduino-rainsensor-module-guide-and-tutorial

[10] Reichen Stein, "Arduino Module Rain Sensor," 2014. [Online]. Available: http://www.instructables.com/id/ArduinoModules-Rain-Sensor/.

[11] TRIKUENI DARMANTO, "No Title," 2014. [Online]. Available: http://trikueni-desainsistem.blogspot.co.id/2014/03/PengertianMotor-Servo.html.

[12] P. P. Specification, "Single chip $2.4 \mathrm{GHz}$ Transceiver," no. March, pp. 1-39, 2006.

[13] L. Kumala, "No Title," 2013. [Online]. Available: https://splashtronic.wordpress.com/2013/10/2 9/modul-wireless-rf-nrf24101/. 
[14] L. D. Resistors, "How it Works Light dependant resistors have many uses, many of the uses A light sensor or LDR can be very easily interfaced with an."

[15] D. Uk, "Temperature Sensor DHT 22 Humidity \& Temperature Sensor," 2010.

[16] R. A. M. Lpddr and B. Bcm, "Raspberry Pi 3 Model B Raspberry Pi 3 Model B."

[17] "Domoticz," 2012. [Online]. Available: https://www.domoticz.com/.

[18] F. Quintero, "MQTT - IoT Messaging Protocol Francisco Quintero Lead Firmware Engineer."

[19] Abhishek Viswanathan, "Analysis of Power Consumption of the MQTT Protocol," 2017.

[20] "Android Studio." [Online]. Available: https://developer.android.com/studio/intro/ind ex.html?hl=id. 